\title{
THE IMPACT OF MATERNAL EMPLOYMENT ON HEALTH OF CHILDREN UNDER TWO YEARS OLD
}

\author{
By \\ Abu salem ME ${ }^{1}$, ALshazaly $\mathrm{HM}^{1}$, Ibrahem RA ${ }^{1}$, \\ Al-oshari SHA ${ }^{2}$ and Allam HK1 \\ ${ }^{1}$ Department of Public Health and Community Medicine, Faculty of Medicine, \\ Menoufia University, Egypt, ${ }^{2}$ Department of Public Health and \\ Community Medicine, Faculty of Medicine, Taiz University, Yemen.
}

Corresponding author: Al-oshari SHA: Email:samar_aloshari@yahoo.com

\begin{abstract}
Introduction: Women's labor force participation has risen around the globe in the past few decades, maternal employment can affect children's well-being through multiple channels, including income effects, decisions about health inputs, and time spent with children. Overall, women's employment affects child health widely. Aim of work: To study the impact of maternal employment on breastfeeding practice, nutritional status, and motor development of children under two years old. Materials and methods: An analytical cross-sectional study was conducted during the period from May 2018 to December 2019, on 400 children under two years old who were attending a Pediatrics Clinic or Vaccination Unit at the Primary Health Care Centers at Shebin Alkom district, Menoufia governorate. The children who fulfilled inclusion criteria were involved in the study, and their mothers were interviewed using an interview questionnaire. Physical examination and complete blood count were done for children. Results: Working mothers represented $25 \%$ of the studied sample. There were statistically significant differences between employed and unemployed mothers as regards education, income, and socioeconomic standard. There were statistically significant differences between the studied groups in breastfeeding practices; exclusive breastfeeding, frequency and duration of breastfeeding were lower among employed mothers, the rate of hospitalization and different infections, i.e., gastroenteritis, otitis media, and repeated throat infection, were significantly higher among children of employed mothers compared to children of unemployed mothers ( $p$-value $<0.05$ ). There was no statistically significant difference between children of employed and unemployed mothers as regard motor development, dentation, weight for age, length for age, and weight for length,
\end{abstract}


while anemia was significantly higher among children of unemployed compared to employed mothers. Conclusion and recommendations: Maternal employment has a negative impact on breastfeeding practice and increase infectious diseases occurrence and hospitalization rate among children. The study showed that there was no effect on motor development and nutrition status of the children. The government has to encourage the implementation of national legislations, which mandated the employers to provide their employees with childcare centers and nurseries near to the workplace.

Keywords: Maternal employment, Child health, Breastfeeding and Nutritional status.

\section{Introduction}

As women participation in the labor market has been rising globally, a growing literature has emerged to explore whether maternal employment is a determinant of child health (Rashad and Sharaf, 2019). Women not only have become engaged in market work, but the nature of employment has also evolved as women have shifted from agriculture into manufacturing and services. In addition, women have entered into non-traditional occupations that were formerly open only to men (Acharya, 2008). Globally, Women's labor force participation rate is $48.5 \%$ (ILO, 2018). In Egypt, women participate in $22.9 \%$ of the total workforce (EDHS, 2015). The direction and magnitude of maternal work's effect on child health are theoretically ambiguous. The most evident mechanism through which maternal employment might positively affect children is through an increase in family income (Currie and Lin, 2007). On the other hand; maternal employment may result in a loss of childcare time as working women have less time to breastfeed their children, preparing rich-nutrition food and take them regularly to health care providers (Majbouri, 2016).

Many factors may be involved in the relationship between breastfeeding and employment of mother such as the timing of return to work, the number of hours worked per week, the availability of a suitable and convenient childcare facility, the location of the work and lack of support from employers, family members and colleagues (Su-Ying, 2013). Women's employment was associated with a decline in the duration of breastfeeding (Kimbero, 2006). The issue regarding whether maternal employment in the labor market results in net benefits for children's nutritional status remains controversial. In closing, developing country studies have found maternal employment to have positive, negative, and no substantial impacts on children's nutritional 
status. Differences across studies in methodology, social context, and the types of work that women perform help to explain this lack of consensus (Meyer, 2016) . Predicting the direction of the relationship between maternal employment and child nutritional status is not apparent (Rashad and Sharaf, 2019). There are still many unanswered questions about the impact of early maternal employment on children's development. The most important are motor and cognitive development and whether maternal employment in the first year of life has any lasting effects on children's cognitive outcomes (Ruhm, 2008).Some of the concern about changes in children's health status when mothers enter the labor market arises from the change in childcare situations, especially when children enter care centers. These concerns include arguments that non-parental care introduces a higher risk that a child may be exposed to infectious diseases or become injured (Baker et al., 2008).

\section{Aim of work}

This study aims to determine the impact of maternal employment on breastfeeding practice, nutritional status and motor development of children under two years old.

\section{Materials and methods}

Study design: It is an analytical cross-sectional study

\section{Place and duration of the study:} The study was carried out in primary health care units in Shebin Alkom district (Menoufia Governorate) during the period from May 2018 to December 2019.

Shebin Alkom district has 12 Primary Health Care Centers and Units distributed at Shebin Alkom city and the surrounding villages. The targeted population was selected from one urban and two rural health centers using simple random sampling. Shebin Alkom city include two Primary Health Care Centers, one selected (Maternal and Child Health center Bahary) by simple random sample as an urban unit. From ten rural health centers, two rural Primary Health Care Centers (Elbatanoun and Shobrabas) were selected by simple random sample.

\section{Study sample}

Sample size: It was calculated by using Epi Info (2000) program at $95 \%$ confidence intervals of and $80 \%$ power of the study, out of the total number of children under two years old in Shebin Al-Kom district were 35200. The 
sample size was calculated to be 380 children, by adding $10 \%$ to total sample size calculated, the final sample size was 420 participants, the response rate was $95.2 \%$, so the total studied children was 400 child.

\section{Inclusion Criteria:}

- Age up to 24 months

- Both sexes were included.

- Apparently healthy children living in Shebin Alkom districts, Menoufia Governorate,

\section{Exclusion Criteria:}

- History of prematurity and malnutrition.

- Children with congenital anomalies.

\section{Study methods:}

All the participants were subjected to:

\section{1- A pre-designed interviewed} questionnaire which included:

a- Age, sex, and residence of the child.

b- Educational and occupational status of the parents.

c- History of breastfeeding, duration of breastfeeding and weaning practices

d- Child medical history e- The questionnaire included items related to motor developmental milestones of the children as age of crawling, sitting, walking and teething etc..........

f- The socioeconomic standard was assessed through their education, occupation, income, the number of individuals per room, type of housing, material possessions, etc........ according to (El-Gilany et al., 2012).

\section{2- Physical examination:}

Each child participated in the study was examined, and the anthropometric measures were taken, including weight that was a record by using an electronic weighing scale; the length of the child and head circumference was measured while the child was lying. Nutrition assessment was done by $\mathrm{Z}$ score, which is a system that expresses the anthropometric value as a number of standard deviations or Z-scores below or above the reference means or median value.

The following growth indicators were used to assess a child nutritional status (WHO, 2008):

g- a- Length-for-Age Z score (HAZ) which was interpreted as : between 
2SD to-2SD= Normal length, below $-2 \mathrm{SD}=$ Stunted and below $-3 \mathrm{SD}=$ Severely stunted

h- b- Weight-for-Age Z score (WAZ) which was interpreted as: between 2SD to-2SD= Normal weight, below -2SD =Underweight and below $-3 \mathrm{SD}=$ Severely underweight

i- c- Weight-for-Length (WLZ) was interpreted as: above $3 \mathrm{SD}=$ Obese, above $2 \mathrm{SD}=$ Overweight, above $1 \mathrm{SD}=$ Possible risk of overweight, below $1 \mathrm{SD}$ to and above $-2 \mathrm{SD}=$ Normal weight, below $-2 \mathrm{SD}=$ Wasted and below $-3 \mathrm{SD}=$ Severely wasted.

Motor development was assessed by milestone Motor Development Chart.

\section{3- Investigation:}

Complete blood count (CBC) was done for children between 6 and 59 months (generally referred to as underfives), the threshold $\mathrm{Hb}$ level for being non-anemic is $11(\mathrm{mg} / \mathrm{dL})(\mathrm{WHO}, 2011)$

\section{Consent}

Formal consent was taken from mothers before being enrolled and after an explanation of the aim, benefits of the study.

\section{Ethical approval}

Medical Ethics Committee at Menoufia Faculty of Medicine approved the study protocol before starting.

\section{Data management}

Data were collected, tabulated, statistically analyzed using an IBM personal computer with IBM Statistical Package of Social Science (SPSS) version 22 (SPSS Inc., Chicago, Illinois, USA). Quantitative data normality was tested using Shapiro-Wilk and KolmogorovSmirnov tests. Student's t-test was used for parametric data, and the Mann-Whitney test for non-parametric Chi-squared test $\left(\chi^{2}\right)$ was used to study the association between two qualitative variables, while Fisher's exact test for $2 \times 2$ tables when expected cell count of more than $25 \%$ of cases was less than 5. A p-value of $<0.05$ was considered statistically significant. 


\section{Results}

Table (1): Comparison of demographic data between employed and unemployed mothers.

\begin{tabular}{|c|c|c|c|c|c|c|}
\hline \multirow[t]{2}{*}{ Demographic data } & \multicolumn{2}{|c|}{$\begin{array}{c}\text { Employed mothers } \\
\text { No=100 }\end{array}$} & \multicolumn{2}{|c|}{$\begin{array}{c}\text { Unemployed mothers } \\
\text { No }=\mathbf{3 0 0}\end{array}$} & \multirow{2}{*}{$\begin{array}{c}\text { Test of } \\
\text { significance }\end{array}$} & \multirow[t]{2}{*}{ p-value } \\
\hline & No & $\%$ & No & $\%$ & & \\
\hline $\begin{array}{l}\text { Age } \\
\text { Mean } \pm \text { SD } \\
\text { Range } \\
\end{array}$ & \multicolumn{2}{|c|}{$\begin{array}{c}29.23 \pm 3.82 \\
20-38 \\
\end{array}$} & \multicolumn{2}{|c|}{$\begin{array}{c}28.68 \pm 4.33 \\
19-38\end{array}$} & $\begin{array}{c}\mathrm{t} \# \\
1.18\end{array}$ & 0.236 \\
\hline $\begin{array}{l}\text { Age of the husband } \\
\text { Mean } \pm \text { SD } \\
\text { Range }\end{array}$ & \multicolumn{2}{|c|}{$\begin{array}{c}34.68 \pm 3.57 \\
28-42 \\
\end{array}$} & \multicolumn{2}{|c|}{$\begin{array}{c}34.16 \pm 4.72 \\
25-43\end{array}$} & $\begin{array}{c}\mathrm{t} \# \\
1.15\end{array}$ & 0.248 \\
\hline $\begin{array}{l}\text { Age of the children in months } \\
\text { Mean } \pm \text { SD } \\
\text { Range }\end{array}$ & \multicolumn{2}{|c|}{$\begin{array}{c}15.95 \pm 6.26 \\
4-24 \\
\end{array}$} & \multicolumn{2}{|c|}{$\begin{array}{c}14.92 \pm 5.45 \\
4-24 \\
\end{array}$} & $\begin{array}{l}\text { U\#\# } \\
1.69\end{array}$ & 0.091 \\
\hline $\begin{array}{l}\text { Sex of the children } \\
\text { Male } \\
\text { Female } \\
\end{array}$ & $\begin{array}{l}40 \\
60 \\
\end{array}$ & $\begin{array}{l}40 \\
60 \\
\end{array}$ & $\begin{array}{l}144 \\
156 \\
\end{array}$ & $\begin{array}{l}48 \\
52 \\
\end{array}$ & $\begin{array}{c}\chi^{2} \\
1.93\end{array}$ & 0.164 \\
\hline $\begin{array}{l}\text { Residence } \\
\text { Urban } \\
\text { Rural }\end{array}$ & $\begin{array}{l}60 \\
40\end{array}$ & $\begin{array}{l}60 \\
40\end{array}$ & $\begin{array}{l}172 \\
128\end{array}$ & $\begin{array}{l}57.4 \\
42.6\end{array}$ & $\begin{array}{c}\chi^{2} \\
0.219\end{array}$ & 0.640 \\
\hline $\begin{array}{l}\text { Education } \\
\text { Low education } \\
\text { High education } \\
\end{array}$ & $\begin{array}{l}20 \\
80\end{array}$ & $\begin{array}{l}20 \\
80\end{array}$ & $\begin{array}{l}164 \\
136 \\
\end{array}$ & $\begin{array}{l}54.6 \\
45.4 \\
\end{array}$ & $\begin{array}{c}\chi^{2} \\
36.29\end{array}$ & $<0.001 * *$ \\
\hline $\begin{array}{l}\text { Husband's education } \\
\text { Low education } \\
\text { High education } \\
\end{array}$ & $\begin{array}{l}28 \\
72 \\
\end{array}$ & $\begin{array}{l}28 \\
72 \\
\end{array}$ & $\begin{array}{c}88 \\
212 \\
\end{array}$ & $\begin{array}{l}29.3 \\
70.7 \\
\end{array}$ & $\begin{array}{c}\chi^{2} \\
0.06 \\
\end{array}$ & 0.799 \\
\hline $\begin{array}{l}\text { Husband's occupation } \\
\text { Unskilled workers } \\
\text { Skilled workers } \\
\text { Employees } \\
\text { Professionals } \\
\end{array}$ & $\begin{array}{l}4 \\
28 \\
56 \\
12 \\
\end{array}$ & $\begin{array}{c}4 \\
28 \\
56 \\
12 \\
\end{array}$ & $\begin{array}{c}28 \\
124 \\
132 \\
16 \\
\end{array}$ & $\begin{array}{c}9.3 \\
41.3 \\
44 \\
5.3 \\
\end{array}$ & $\begin{array}{c}\chi^{2} \\
13.23\end{array}$ & $0.004 *$ \\
\hline $\begin{array}{l}\text { Number of children } \\
1-2 \\
3-4 \\
\text { More than } 4 \\
\end{array}$ & $\begin{array}{c}68 \\
32 \\
0 \\
\end{array}$ & $\begin{array}{c}68 \\
32 \\
0 \\
\end{array}$ & $\begin{array}{c}180 \\
112 \\
8 \\
\end{array}$ & $\begin{array}{c}60 \\
37.3 \\
2.7 \\
\end{array}$ & $\begin{array}{c}\chi^{2} \\
4.033\end{array}$ & 0.133 \\
\hline $\begin{array}{l}\text { Income } \\
\text { Not Sufficient } \\
\text { Sufficient } \\
\text { More than sufficient } \\
\end{array}$ & $\begin{array}{l}36 \\
40 \\
24 \\
\end{array}$ & $\begin{array}{l}36 \\
40 \\
24 \\
\end{array}$ & $\begin{array}{c}128 \\
156 \\
16 \\
\end{array}$ & $\begin{array}{c}42.6 \\
52 \\
5.4 \\
\end{array}$ & $\begin{array}{c}\chi^{2} \\
29.15\end{array}$ & $<0.001 * *$ \\
\hline $\begin{array}{l}\text { Socioeconomic standard } \\
\text { Low } \\
\text { Moderate } \\
\text { High }\end{array}$ & $\begin{array}{l}24 \\
52 \\
24\end{array}$ & $\begin{array}{l}24 \\
52 \\
24\end{array}$ & $\begin{array}{c}116 \\
170 \\
14\end{array}$ & $\begin{array}{c}38.7 \\
56.7 \\
4.6\end{array}$ & $\begin{array}{c}\chi^{2} \\
23.28\end{array}$ & $<0.001 * *$ \\
\hline
\end{tabular}

$\chi^{2}$ Chi-Square test

*: Significant
$\# \mathrm{t}=$ student $\mathrm{t}$-test

**: Highly significant
\#\# U=Mann-Whitney test 
Table 1 showed that there was statistically significant difference between employed and unemployed mothers as regards education, income, and socioeconomic standard (p-value <0.05)

Table (2): Comparison of feeding practices between employed and unemployed mothers.

\begin{tabular}{|c|c|c|c|c|c|c|}
\hline & \multicolumn{2}{|c|}{$\begin{array}{c}\text { Employed } \\
\text { mothers } \\
\text { No=100 }\end{array}$} & \multicolumn{2}{|c|}{$\begin{array}{c}\text { Unemployed } \\
\text { mothers } \\
\text { No }=\mathbf{3 0 0}\end{array}$} & \multirow[t]{2}{*}{ Test of } & \multirow[t]{2}{*}{ p-value } \\
\hline & No & $\%$ & No & $\%$ & & \\
\hline $\begin{array}{l}\text { Start breastfeeding directly } \\
\text { after labor } \\
\text { Yes } \\
\text { NO }\end{array}$ & $\begin{array}{c}97 \\
3\end{array}$ & $\begin{array}{c}97 \\
3\end{array}$ & $\begin{array}{c}293 \\
7\end{array}$ & $\begin{array}{c}97.7 \\
2.3\end{array}$ & $\begin{array}{l}\text { \#FXT } \\
0.137\end{array}$ & 0.716 \\
\hline $\begin{array}{l}\text { Exclusive breastfeeding in the } \\
\text { first six months }(\mathbf{N o}=\mathbf{3 9 0}) \\
\text { Yes } \\
\text { NO }\end{array}$ & $\begin{array}{l}32 \\
65\end{array}$ & $\begin{array}{l}32.9 \\
67.1\end{array}$ & $\begin{array}{l}190 \\
103\end{array}$ & $\begin{array}{l}64.8 \\
35.2\end{array}$ & $\begin{array}{c}\chi^{2} \\
30.16\end{array}$ & \\
\hline $\begin{array}{l}\text { Number of breastfeeding per } \\
\text { day(No=390) } \\
<\text { five } \\
\text { Five to eight } \\
>\text { eight }\end{array}$ & $\begin{array}{l}36 \\
37 \\
24 \\
\end{array}$ & $\begin{array}{l}37.1 \\
38.1 \\
24.8 \\
\end{array}$ & $\begin{array}{c}54 \\
127 \\
112 \\
\end{array}$ & $\begin{array}{l}18.4 \\
43.3 \\
38.2 \\
\end{array}$ & 15.29 & \\
\hline $\begin{array}{l}\text { Timing of breastfeeding } \\
\mathbf{( N o = 3 9 0 )} \\
\text { Every } 4 \text { hours } \\
\text { Every } 6 \text { hours } \\
\text { When baby needs }\end{array}$ & $\begin{array}{l}22 \\
50 \\
25\end{array}$ & $\begin{array}{l}22.7 \\
51.5 \\
25.8\end{array}$ & $\begin{array}{l}55 \\
111 \\
127\end{array}$ & $\begin{array}{l}18.8 \\
37.9 \\
43.3\end{array}$ & $\begin{array}{c}\chi^{2} \\
9.63\end{array}$ & $0.008 *$ \\
\hline $\begin{array}{l}\text { Child }>\text { s age at cessation of } \\
\text { breastfeeding in months } \\
\text { Mean } \pm \mathrm{SD} \\
\text { Range }\end{array}$ & \multicolumn{2}{|c|}{$\begin{array}{c}7.27 \pm 2.84 \\
4-12\end{array}$} & \multicolumn{2}{|c|}{$\begin{array}{c}13.82 \pm 2.65 \\
7-18\end{array}$} & $\begin{array}{l}\text { U\#\# } \\
9.83\end{array}$ & \\
\hline $\begin{array}{l}x^{2} \text { Chi-Square test } \\
\text { : Significant }\end{array}$ & Hig & $\begin{array}{l}\text { exa } \\
\text { igni }\end{array}$ & & & ann- & ney test \\
\hline
\end{tabular}


Table 2 showed that there was no statistically significant difference between employed and unemployed mothers as regards the start of breastfeeding after labor. There were statistically significant differences between employed and unemployed mothers in exclusive breastfeeding in the first six months of children's age, number of breastfeeding/day, and breastfeeding timing (p-value <0.05). Employed mothers stop breastfeeding earlier than unemployed mothers.

Table (3): Children's medical history of employed and unemployed mothers.

\begin{tabular}{|c|c|c|c|c|c|c|}
\hline & \multicolumn{2}{|c|}{\begin{tabular}{|c|}
$\begin{array}{c}\text { Employed mothers } \\
\text { No }=100\end{array}$ \\
\end{tabular}} & \multicolumn{2}{|c|}{\begin{tabular}{|c} 
Unemployed mothers \\
No $=300$
\end{tabular}} & \multirow[t]{2}{*}{$\chi^{2}$} & \multirow{2}{*}{ p-value } \\
\hline & No & $\%$ & No & $\%$ & & \\
\hline $\begin{array}{l}\text { History of hospitalization } \\
\text { Yes } \\
\text { NO }\end{array}$ & $\begin{array}{l}16 \\
84\end{array}$ & $\begin{array}{l}16 \\
84\end{array}$ & $\begin{array}{c}4 \\
296\end{array}$ & $\begin{array}{c}1.3 \\
98.7\end{array}$ & 33.96 & $<0.001 * *$ \\
\hline $\begin{array}{l}\text { History of gastroenteritis } \\
\text { Yes } \\
\text { NO }\end{array}$ & $\begin{array}{l}45 \\
55\end{array}$ & $\begin{array}{l}45 \\
55\end{array}$ & $\begin{array}{c}44 \\
256\end{array}$ & $\begin{array}{l}14.7 \\
85.3\end{array}$ & 39.89 & $<0.001 * *$ \\
\hline $\begin{array}{l}\text { History repeated throat } \\
\text { infection } \\
\text { Yes } \\
\text { NO }\end{array}$ & $\begin{array}{l}64 \\
36\end{array}$ & $\begin{array}{l}64 \\
36\end{array}$ & $\begin{array}{l}100 \\
200\end{array}$ & $\begin{array}{l}33.3 \\
66.7\end{array}$ & 29.15 & $<0.001 * *$ \\
\hline $\begin{array}{l}\text { History of otitis media } \\
\text { Yes } \\
\text { No }\end{array}$ & $\begin{array}{l}20 \\
80\end{array}$ & $\begin{array}{l}20 \\
80\end{array}$ & $\begin{array}{c}32 \\
268\end{array}$ & $\begin{array}{l}10.7 \\
89.3\end{array}$ & 5.77 & $0.016 *$ \\
\hline $\begin{array}{l}\text { History of anemia } \\
\text { Yes } \\
\text { NO }\end{array}$ & $\begin{array}{c}3 \\
97\end{array}$ & $\begin{array}{c}3 \\
97\end{array}$ & $\begin{array}{c}24 \\
276\end{array}$ & $\begin{array}{c}8 \\
92\end{array}$ & 2.97 & 0.084 \\
\hline
\end{tabular}

$\chi^{2}=$ Chi-Square test

*: Significant

**: Highly significant

Table 3 showed that the history of different infections and hospitalization rate were statistically significantly higher among children of employed mothers compared to children of unemployed mothers ( $p$-value $<0.05$ ). Anemia was higher among children of unemployed mothers compared to children of employed mothers but not to a statistical significance level. 
Table (4): Comparison of children motor development and dentation between employed and unemployed mothers.

\begin{tabular}{|c|c|c|c|c|c|c|}
\hline & \multicolumn{2}{|c|}{$\begin{array}{c}\text { Employed } \\
\text { mothers } \\
\text { No=100 }\end{array}$} & \multicolumn{2}{|c|}{$\begin{array}{c}\text { Unemployed } \\
\text { mothers } \\
\text { No }=\mathbf{3 0 0}\end{array}$} & $\begin{array}{c}\text { Test of } \\
\text { significance }\end{array}$ & p-value \\
\hline Age of crawling & \multirow{2}{*}{\multicolumn{2}{|c|}{$8.14 \pm 1.46$}} & \multirow{2}{*}{\multicolumn{2}{|c|}{$8.15 \pm 0.99$}} & $\mathrm{t} \#$ & \\
\hline Mean \pm SD & & & & & 0.081 & 0.936 \\
\hline Range & \multicolumn{2}{|c|}{$5-10$} & \multicolumn{2}{|c|}{$5-11$} & & \\
\hline Age of sitting & \multirow{3}{*}{\multicolumn{2}{|c|}{$\begin{array}{c}9.78 \pm 1.20 \\
7-12\end{array}$}} & \multirow{3}{*}{\multicolumn{2}{|c|}{$\begin{array}{c}9.90 \pm 1.80 \\
7-12\end{array}$}} & $\mathrm{t} \#$ & \\
\hline Mean \pm SD & & & & & & 0.481 \\
\hline Range & & & & & 0.707 & \\
\hline Age of walking & \multirow{3}{*}{\multicolumn{2}{|c|}{$\begin{array}{c}12.92 \pm 1.77 \\
10-18\end{array}$}} & \multirow{3}{*}{\multicolumn{2}{|c|}{$\begin{array}{c}12.89 \pm 1.83 \\
9-18\end{array}$}} & $\mathrm{t} \#$ & \\
\hline Mean \pm SD & & & & & & 0.902 \\
\hline Range & & & & & 0.123 & \\
\hline \multirow{4}{*}{$\begin{array}{l}\text { Age of dentation } \\
\text { Mean } \pm \text { SD } \\
\text { Range }\end{array}$} & \multirow{3}{*}{\multicolumn{2}{|c|}{$\begin{array}{c}8.07 \pm 2.49 \\
6-14\end{array}$}} & \multirow{3}{*}{\multicolumn{2}{|c|}{$\begin{array}{c}7.54 \pm 1.75 \\
5-14\end{array}$}} & $\mathrm{t} \#$ & \multirow{4}{*}{0.074} \\
\hline & & & & & \multirow{3}{*}{1.80} & \\
\hline & & & & & & \\
\hline & No & $\%$ & No & $\%$ & & \\
\hline Motor development & & & & & FXT\#\# & \\
\hline Normal & 100 & 100 & 299 & 99.7 & 0.334 & 0.563 \\
\hline Delayed & 0 & 0 & 1 & 0.3 & & \\
\hline Dentation & & & & & $\chi^{2}$ & \\
\hline Normal & 89 & 89 & 249 & 83 & & 0.185 \\
\hline Delayed & 11 & 11 & 51 & 17 & 1.75 & \\
\hline
\end{tabular}

$\chi^{2}=$ Chi-Square test

\# $\mathrm{t}=$ students t-test

\#\#FXT=Fishers exact test

Table 4 showed that there was no statistically significant difference between children of employed and unemployed mothers as regards age of crawling, sitting, walking and dentation ( $\mathrm{p}$-value $>0.05$ ) 
Table (5): Comparison of nutritional status with a $\mathbf{Z}$ score of employed and unemployed mother's children.

\begin{tabular}{|c|c|c|c|c|c|c|}
\hline & \multicolumn{2}{|c|}{$\begin{array}{c}\text { Employed mothers } \\
\text { No }=100\end{array}$} & \multicolumn{2}{|c|}{$\begin{array}{c}\text { Unemployed mothers } \\
\text { No }=\mathbf{3 0 0}\end{array}$} & \multirow[t]{2}{*}{$\chi^{2}$} & \multirow[t]{2}{*}{ p-value } \\
\hline & No & $\%$ & No & $\%$ & & \\
\hline $\begin{array}{l}\text { Weight for age } \\
\text { Normal weight } \\
\text { Underweight }\end{array}$ & $\begin{array}{c}92 \\
8\end{array}$ & $\begin{array}{c}92 \\
8\end{array}$ & $\begin{array}{c}268 \\
32\end{array}$ & $\begin{array}{l}89.3 \\
10.7\end{array}$ & 0.593 & 0.441 \\
\hline $\begin{array}{l}\text { Length for age } \\
\text { Normal length } \\
\text { Stunted }\end{array}$ & $\begin{array}{c}96 \\
4\end{array}$ & $\begin{array}{c}96 \\
4\end{array}$ & $\begin{array}{c}287 \\
13\end{array}$ & $\begin{array}{c}95.7 \\
4.3\end{array}$ & 0.020 & 0.886 \\
\hline $\begin{array}{l}\text { Weight for length } \\
\text { Obese +over weight }+ \text { risk for } \\
\text { overweight } \\
\text { Normal weight } \\
\text { Wasted }\end{array}$ & $\begin{array}{c}8 \\
90 \\
2\end{array}$ & $\begin{array}{c}8 \\
90 \\
2\end{array}$ & $\begin{array}{c}8 \\
282 \\
10\end{array}$ & $\begin{array}{l}2.6 \\
94 \\
3.3\end{array}$ & 5.91 & 0.062 \\
\hline $\begin{array}{l}\text { Anemia } \\
\text { Yes } \\
\text { NO } \\
\end{array}$ & $\begin{array}{l}11 \\
89 \\
\end{array}$ & $\begin{array}{l}11 \\
89 \\
\end{array}$ & $\begin{array}{c}61 \\
239 \\
\end{array}$ & $\begin{array}{l}20.3 \\
79.7 \\
\end{array}$ & 4.42 & 0.035* \\
\hline
\end{tabular}

$\chi^{2}=$ Chi-Square test

*: Significant

Table 5 showed that there was no statistically significant difference between children of employed and unemployed mothers using $\mathrm{Z}$ score as regards weight for age, length for age and weight for length, while anemia was significantly higher among children of unemployed compared to employed mothers ( $p$-value $<0.05$ ).

\section{Discussion}

Two different mechanisms on the effects of mother work on children's health have been postulated; first mother's work increase family income, second, the time taken away from childcare when she goes to work. The first one is direct and positive the other is inverse and negative (Currie and Lin, 2007). The magnitude of maternal work's effect on child health is still not clear. We did this study in a trial to clarify the impacts of mother's work on their child's health.

The current study showed that the percentage of working mothers of the studied group was $25 \%$ (Table 1). This result was in line with EDHS, 2015, which reported that women participate in $22.9 \%$ of the total workforce in Egypt. 
The present study demonstrated that the educational level was statistically higher among employed compared to unemployed mothers (Table 1). This difference can be explained by the fact that women with better education had better work chance. This was in agreement with Carlijn et al., 2017, who reported that there is a link between women education and employment. Our study found that employed mothers had higher income and better socioeconomic standard than unemployed mothers (Table 1) .This can be explained by that maternal employment increases family income and welfare. This finding comes in agreement with Rashad and Sharaf, 2019. The same findings were approved by Ying-ping et al., 2018 in their study on maternal employment and family socioeconomic status among Taiwanese families.

In the present study, we found that there was no statistically significant difference between employed and unemployed mothers as regards the start of breastfeeding after labor (Table 2 ). This result was in accordance with Tadesse et al., 2019 in their study on exclusive breastfeeding and maternal employment among mothers of infants from three to five months old in the
Fafan zone, Somali regional state of Ethiopia. Also Hawkins et al., 2007 detected the same results in their study on maternal employment and breastfeeding initiation: findings from the Millennium Cohort Study.

There were statistically significant differences between employed and unemployed mothers in exclusively breastfeeding (EBF) in first six months (Table 2) as 32\% of employed mothers exclusively breastfeeding versus $56.9 \%$ of unemployed mothers this may be explained by the need of employed mothers to use additional types of feeding during their absence at work. This negative impact of maternal employment on EBF was found in different studies: Shaheen et al., 2018 in their study about the barriers to breastfeeding among women and the study done by Al-Sahab et al., 2010 on the prevalence and predictors of 6-month exclusive breastfeeding among Canadian women: a national survey.

The current study showed that there was a statistically significant difference in the number of breastfeeds/day, as $37.1 \%$ of employed mothers and $18.4 \%$ of unemployed mother's breastfed less than five times/day (Table 2). This result comes in accordance with Farahat et al., 
2007, who reported that $30 \%$ and $11 \%$ of employed and unemployed mothers' breastfed less than five times per day. There was a statistically significant difference as regards the age of children when cessation breastfeeding, where employed mothers stop breastfeeding earlier than unemployed mothers (Table 2). This result was consistent with Rivera-Pasquel et al., 2015 in their study about breast-feeding and maternal employment in Mexico and the study done by Cooklin, 2008 about maternal employment and breast feeding: longitudinal study of Australian children. These adverse effects of maternal employment on breastfeeding practices may be attributed to the lack of breastfeedingfriendly work environment, early return to work, and long work hours, which lead to infrequent or widely spaced feeding that will eventually lead to drop of the amount of milk produced and finally cessation of lactation.

The present study revealed that the history of different infectious diseases was significantly higher among children of employed mothers compared to children of unemployed (Table 3 ). This may be because children of employed mothers spend more time in nurseries and childcare centers; care outside the home usually increases children's direct exposure to potentially infectious agents and, therefore, the risk of having an infectious diseases. This result was consistent with Gordon et al., 2007 who found that maternal employment has statistically significant direct effects on the incidence of infectious diseases and agreed with Morrill, 2011who reported that maternal employment increases the probability a child has episodes of intestinal and respiratory infections. The rate of hospitalization was significantly higher among children of employed mothers than children of unemployed mothers (Table 3). This result was in line with Morrill, 2011.

We found that there was no statistically significant difference between children of employed and unemployed mothers as regards age of crawling, sitting, walking and dentations (Table 4), These results have disagreed with that of Koutra et al., 2012 in their study on sociodemographic determinants of infant neurodevelopment at 18 months of age: Mother-child cohort study in Crete, Greece.

The present work showed that maternal employment has no effect 
on children nutritional status (Table 5). This result was agreed with Hiwot et al., 2017 and Ferguson et al., 2017, who showed that maternal employment does not have a marked impact on the growth and nutrition status of children. However, different results were noted by McDonnell, 2014, and Fitzsimons and pongiglione, 2019, who found that children of employed mothers have an increased risk of childhood overweight and increases children's BMI (Body Mass Index). On the other hand, Yana van der, 2016 reported that maternal employment was associated with a greater risk of stunting and wasting. The same association was noted by Baharudin et al., 2019 and Rashad and Sharaf, 2019 who found a strong negative association between maternal employment and child nutritional status as maternal employment deteriorate children HAZ (Height for Age Z score). We found that anemia was significantly higher among children of unemployed than employed mothers, $20.3 \%$ versus $11 \%$ (Table 5). This can be explained by high income, education level and socioeconomic status of employed than unemployed mothers.

\section{Conclusion and recommendations:}

Maternal employment has a negative impact on breastfeeding practice and increase infectious diseases occurrence and hospitalization rate among children. The study also showed that there was no effect between maternal employment and the motor development and nutrition status of the children. The government has to encourage the implementation of national legislations, which mandated the employers to provide their employees with childcare centers and nurseries near to the workplace.

\section{Funding}

None

\section{Conflict of interest}

None declared

\section{Acknowledgment}

The authors would like to thank the participants who generously agreed to participate in this work for their time to provide valuable information.

\section{References}

1. Acharya S (2008): Poverty Alleviation and the Industrial Employment of Women (The Case of Nepal). J Int dev; 20 (5): 670-85.

2. Al-Sahab B, Lanes A, Feldman M and Tamim H: (2010): Prevalence and predictors of 6-month exclusive breastfeeding among Canadian women: a national survey. BMC Pediatrics; 10:20.

3. Baharudin A, Man CS, Ahmad M, Wong N, Salleh R, et al.(2019): Associated Factors to Prevalence of Childhood under 
Nutrition in Malaysia: Findings from the National Health and Morbidity Survey (NHMS 2016).Health Science J ; 13(3):19

4. Baker M, Jonathan $\mathrm{G}$, and Kevin M (2008): Universal Child Care, Maternal Labor Supply, and Family Well-Being. J Polit Econ ; 116 (4):709-45

5. Carlijn B, Kars O, Gerbert K and Niels S (2017): Women s Worldwide Educationemployment Connection: A Multilevel Analysis of the Moderating Impact of Economic Political, and Cultural Contexts. World Development; 99:41.

6. Coolin AR (2008): Maternal employment and breast feeding: results from the longitudinal study of Australian children .Pediatrics /Acta Pediatric; 97:620-3.

7. Currie J and Lin W (2007): Chipping away at health: More on the relationship between income and child health .Health Affairs; 26(2):331-44.

8. EDHS (Egypt Demographic and Health Survey) (2015): Ministry of Health and Population Cairo, Egypt and El-Zanaty and Associates Cairo, Egypt. Available at; http://ghdx.healthdata.org/record/ egypt-special-demographic-and-healthsurvey-2015.

9. El-Gillany A, El-Wehady A and El-Wasify $M$ (2012): updating and validation of the socioeconomic status scale for health research in Egypt. EMHJ; 18:9.

10. Farahat MT, Musaiger AO, Deeb M, Melky A and Salamaa A (2007):Breast Feeding in Employed Mothers "Master thesis" ,Family Medicine department, Faculty of Medicine, Menoufia University.

11. Ferguson A, Penney R and Solo-Gabriele $H$ (2017): A Review of the Field on Children's Exposure to Environmental Contaminants: A Risk Assessment Approach. Int J Environ Res $\mathrm{Pu} ; 14(3)$ :
265-75.

12. Fitzsimons E and Pongiglione B (2019): The impact of maternal employment on children's weight: Evidence from the UK. SSM Popul Health J; 7(3):32-7.

13. Gordon RA, Kaestner R and Korenman S (2007): The effects of maternal employment on child injuries and infectious disease. Demography ; 44(2):307-33

14. Hawkins S, Griffiths LJ, Dezateux C and Law C (2007): Maternal employment and breastfeeding initiation: findings from the Millennium Cohort Study. Pediatr Perinat Epidemiol ;21(3):242-7

15. Hiwot E, Yewelsew A, Eskindir L, Teklemichael $G$ and Tesfalem $T$ (2017): Nutritional status and effect of maternal employment among children aged 6-59 months in Wolayta Sodo Town, Southern Ethiopia: a cross-sectional study. Ethiop J health Sciences; 27(2):155-62

16. ILO (International Labour Organization) (2018): World Employment and Social Outlook: Trends for Women 2018 - Global snapshot: Report, Geneva. https://www.ilo. org/global/research/global-reports/weso/ trends-for-women2018/lang--en/index. htm

17. Kimbro R (2006): On-the-job moms: Work and breastfeeding initiation and duration for a sample of low-income women". Matern Child Hlth J; 10(1):19-29.

18. Kouta K, Leda C, Theano R, Maria V and Eirini G (2012): Socio-demographic determinants of infant neurodevelopment at 18 months of age :Mother-child cohort (Rhea study) in Crete, Greece. Infant Behave Dev; 35(1):48-59.

19. Majbouri M (2016): Against the wind: labor force participation of women and economic instability in Iran .Fem Econ; 22(4):31-53.

20. McDonnell T (2014): Maternal 
employment, childcare and childhood overweight during infancy. UCD School of Economics, University College Dublin. http://www.ucd.ie/geary/ static/publications/workingpapers/ gearywp201411.pdf

21. Meyer S C (2016): Maternal employment and childhood overweight in Germany. Econ Hum Biol ; 23:84-102

22. Morrill M (2011): The Effects of Maternal Employment on the Health of SchoolAge Children. J Health Econ; 30 (2):240 57.

23. Rashad A and Sharaf M (2019): Does Maternal Employment Affect Child Nutritional status? New Evidence from Egypt .Oxford development studies J ; 47(1): 48-62.

24. Rivera-Pasquel M, Escobar L and Gonzalez $T$ (2015): Breast feeding and Maternal Employment: Results from Three National Nutritional survey in Mexico. Matern Child Hlth J;19:1162-72 .

25. Ruhm C (2008): Maternal employment and child development. Handbook of Families and Work (331-354). Lanham, Maryland: University Press of America.

26. Shaheen $\mathrm{H}$, Hegazya $\mathrm{N}$ and Sakrb $\mathrm{S}$ (2018): The barriers to breastfeeding among women: a single-center experience. MMJ ; 31:855-61.

27. Su-Ying Tsai (2013):Impact of BreastFeeding Friendly workplace on an Employed mother's Intention to continue Breast Feeding After Returning to Work .Journal of Breast feeding;8(2):210-6.

28. Tadesse F, Alemayehu Y, Shine S and
Henok A (2019): Exclusive breastfeeding and maternal employment among mothers of infants from three to five months old in the Fafan zone, Somali regional state of Ethiopia: a comparative cross-sectional study. BMC Public Health; 19:10-5.

29. WHO (World Health Organization) (2008): Training Course on Child Growth Assessment.Geneva.https://www.who.int/ childgrowth/training/module_h_directors_ guide.pdf

30. WHO (World Health Organization) (2011): Haemoglobin concentrations for the diagnosis of anaemia and assessment of severity. Vitamin and Mineral Nutrition Information System. Geneva

https://www.academia.edu/4215770/ WHO._Haemoglobin_concentrations for_the_diagnosis_of_anaemia_and assessment_of_severity._Vitamin and_Mineral_Nutrition_Information System._Geneva_World_Health Organization_2011_WHO_NMH_NHD_ MNM_11.1

31. Yana VR (2016): Maternal employment and child health. Book via Qatar National Library (QNL).

32. Ying-ping TT, Li TK and Qing Z (2018): Maternal Employment and Family Socioeconomic Status: Unique Relations to Maternal Stress, Parenting Beliefs, and Preschoolers Adjustment in Taiwanese Families. J Child Fam Stud; 18 (3):8995. 
